\title{
Olfactory fMRI in patients with Parkinson's disease
}

\author{
Thomas Hummel ${ }^{\text {*t}}$, Klaus Fliessbach ${ }^{2+}$, Michael Abele ${ }^{3}$,Thorsten Okulla ${ }^{3}$, Jens Reden ${ }^{1}$, Heinz Reichmann ${ }^{4}$, \\ Ullrich Wüllner ${ }^{3}$ and Antje Haehner ${ }^{1,4}$
}

Smell and Taste Clinic, Department of Otorhinolaryngology, University of Dresden Medical School, Dresden, Germany

2 Department of Epileptology, University of Bonn, Bonn, Germany

${ }^{3}$ Department of Neurology, University of Bonn, Bonn, Germany

${ }^{4}$ Department of Neurology, University of Dresden Medical School, Dresden, Germany

\section{Edited by:}

Sidney A. Simon, Duke University, USA

Reviewed by:

Antje Welge-Lüssen, University

Hospital Basel, Switzerland

Bogdan Draganski, University

Lausanne, Switzerland

${ }^{*}$ Correspondence:

Thomas Hummel, Smell and Taste

Clinic, Department of

Otorhinolaryngology, University of

Dresden Medical School, Fetscherstr.

74, 01307 Dresden, Germany.

e-mail: thummel@mail.zih.tu-dresden.de

tThomas Hummel and Klaus Fliessbach contributed equally.
Hyposmia is one of the early signs in idiopathic Parkinson's disease (PD). Olfactory stimuli were applied during $\mathrm{fMRI}$ scanning to show disease-related modulation of central nervous system structures and to advance our understanding of olfactory dysfunction in PD patients. All participants received either unpleasant stimuli that smelled like rotten eggs or pleasant ones that smelled like roses. Using a block design at a 1.5T scanner we investigated a total of 8 PD patients (mean age $60 \pm 10.9$ years) and 13 age matched controls (mean age $58 \pm 9.6$ years). PD duration ranged from 1 to 9 years (mean 6.63 years); patients had an average "Unified Parkinson's Disease Rating Scale III" score of 23.25 (range, 6-46). Olfactory function was established using the "Sniffin' Sticks" test battery. Patients tended to rate the stimuli presented during fMRI scans as less intense, but also as more pleasant than controls. $f M R I$ results revealed differences between PD patients and controls which depended on the type of stimulation. While both pleasant and unpleasant stimulation was associated with lower activation in the amygdalo-hippocampal complex in patients compared to controls, increased activity in response to pleasant stimuli was observed in the striatum and the left inferior frontal gyrus. In contrast, unpleasant stimulation led to hypoactivation of the ventral striatum in patients (but not in controls) and did not enhance left inferior frontal activity. These results may partly reflect differences between PD patients and healthy controls in the processing of primary dimensions of odors, intensity, and valence.

Keywords: smell, neurodegeneration, olfactory loss, hyposmia, imaging

\section{INTRODUCTION}

There is convincing evidence from numerous studies using both psychophysical (Ramaker et al., 2002; Litvan et al., 2003) and electrophysiological approaches (Hummel et al., 1993; Barz et al., 1997; Hawkes and Shephard, 1998) that olfaction is markedly reduced in idiopathic Parkinson's disease (PD). The decreased olfactory function precedes the onset of motor symptoms by approximately 4 years (Berendse and Ponsen, 2006; Haehner et al., 2007); it appears independent of the medication used and does not respond to changes in medication (Doty et al., 1988). Thus, although hyposmia has already become a useful diagnostic criterium for the presence of idiopathic PD (Doty et al., 1995; Hummel et al., 2010), little is known, however, whether and how the pattern of olfactory activation differs in PD compared to healthy individuals. Westermann et al. (2008) suggested that selective impairment of amygdala and hippocampal activation contributes to olfactory dysfunction in PD. In a functional magnetic resonance imaging (fMRI) study (Westermann et al., 2008), they observed a mainly lateralized activation pattern in PD and discussed the involvement of compensatory mechanisms in the olfactory deficit. In that study, only the pleasant rose-like odor PEA was used for olfactory stimulation which may have biased the pattern of activation. In fact, it is still an open question whether affective experience modulates olfactory activation in individuals with smell dysfunction.

The issue of hedonic-specific activation was addressed in this investigation. We performed fMRI in PD patients and healthy controls while two specific olfactory stimuli, one pleasant (rose-like) and one unpleasant (rotten eggs), were delivered using an olfactometer (OM6b; Burghart instruments, Wedel, Germany). Olfactory stimuli were applied during fMRI scanning to study disease-related modulation of central nervous system structures and to advance our understanding of olfactory dysfunction in PD patients.

\section{MATERIALS AND METHODS SUBJECTS}

Only women were included in order to control for possible sexrelated differences in olfactory abilities. We investigated 11 patients with Parkinson's disease (mean age $60 \pm 10.9$ years) and 13 age matched controls (mean age $58 \pm 9.6$ years). However, three PD patients had to be excluded from the study because they failed to cooperate throughout the entire scanning session. PD duration ranged from 1 to 9 years (mean 6.63 years). Patients presented with a mean "Unified Parkinson's Disease Rating Scale III" (UPDRS III) (Fahn and Elton, 1987) score of 23.3 (range, 6-46). United Kingdom Parkinson's Disease Society Brain Bank Diagnostic Criteria for Parkinson's Disease (Daniel and Lees, 1993) were applied for all patients. None of the control subjects had a history of neurological or psychiatric disease and all subjects were non-smokers. Apart from one PD patient none of the participants exhibited signs of major depression (Beck Depression Inventory, Beck et al., 1961; Hautzinger et al., 1995, BDI score $\geq 15$ ). The study was approved by the local ethics committee and all subjects gave written informed consent. 


\section{OLFACTORY TESTING}

All participants underwent a standardized psychophysical olfactory test, the "Sniffin' Sticks" (Hummel et al., 1997). Odorants were presented in pen-like odor dispensing devices. For odor presentation the pen's cap was removed by the experimenter for approximately $3 \mathrm{~s}$; then the pen's tip was placed approximately $2 \mathrm{~cm}$ in front of both nostrils (Kobal et al., 1996). Testing was performed bilaterally. It involved tests for odor threshold, discrimination, and identification (duration of testing was approximately $30 \mathrm{~min}$ ). Odor thresholds for PEA were assessed using a single-staircase, three-alternative forced-choice procedure. Sixteen dilutions were prepared in a geometric series starting from pure 4\% PEA (dilution ratio 1:2 in propylene glycol) (Croy et al., 2009). Three pens were presented in a randomized order, with two containing the solvent and the third the odorant at a certain dilution. The subject's task was to identify the odor-containing pen. Reversal of the staircase was triggered when the odor was correctly identified in two successive trials for a total of seven reversals. Threshold was defined as the mean of the last four staircase reversal points. Subjects' scores ranged between 1 and 16. In the odor discrimination task, triplets of pens were presented in a randomized order, with two containing the same odorant and the third, a different odorant. Using a three-alternative forced-choice technique, subjects had to determine which of three odor-containing pens smelled differently. A total of 16 triplets were tested. When measuring odor thresholds and odor discrimination, subjects were blindfolded to prevent visual identification of some of the odorant-containing pens. Odor identification was assessed by means of 16 common odors. Using a multiple forced-choice design, identification of individual odors was performed using a list of four descriptors. Again, the subjects' scores ranged from 0 to 16 (for details see Hummel et al., 2007).

Results of the three subtests were presented as a composite "TDI score" (range 1-48) which was the sum of the results obtained for threshold, discrimination, and identification measures. Using this measure, olfactory abilities can be classified in terms of functional anosmia $(<16)$, hyposmia, and normosmia (Hummel et al., 2007).

\section{fMRI ACTIVATION TASK}

We used a two-factorial design with one between-subject factor, "group" (patients vs. controls), and one within-subject factor, "odor". Each subject participated in two sessions with two very common, but hedonically very different olfactory stimuli (phenyl ethylalcohol, PEA, a pleasant rose-like odor; hydrogen sulfide, $\mathrm{H}_{2} \mathrm{~S}$, the unpleasant smell of rotten eggs) which were presented simultaneously to the right and left nostril. Odors were presented intranasally (inner diameter of the Teflon ${ }^{\mathrm{TM}}$ tubing $4 \mathrm{~mm}$ ) using a Burghart OM6b olfactometer (Burghart, Wedel, Germany). To avoid mechanical stimulation the odor pulses were embedded in a constant flow of odorless, humidified air. Stimulus pulses had a duration of $1 \mathrm{~s}$, the interval between stimuli was $2 \mathrm{~s}$; total flow was $8 \mathrm{l} / \mathrm{min}$ with stimuli being presented to both nostrils. Stimulation was administered in blocks of 10 events (i.e., over $28 \mathrm{~s}$ ) followed by a rest period of $32 \mathrm{~s}$ without stimulation. For each condition a session consisted of six stimulation periods each followed by a rest period yielding a duration of approximately $6 \mathrm{~min}$.

\section{fMRI - DATA ACQUISITION}

Scanning was performed on a $1.5 \mathrm{~T}$ Avanto Scanner (Siemens, Erlangen, Germany) using a standard eight-channel head coil. During each of the three sessions, 130 axial echo planar imaging (EPI) scans were acquired during each session including six dummy scans. Scan parameters were: number of slices: 35 ; slice thickness: $3 \mathrm{~mm}$; interslice gap $0.3 \mathrm{~mm}$; matrix size: $64 \times 64$; field of view: $192 \mathrm{~mm}$; echo time (TE): $35 \mathrm{~ms}$; repetition time (TR): $3 \mathrm{~s}$. Slices were aligned parallel to the line between the anterior and the posterior commissure and were set to cover all of the brain and the upper part of the cerebellum. After the experimental session structural T1-weighted images were obtained using a magnet prepared rapid gradient echo (MP-RAGE) sequence at a resolution of $1 \times 1 \times 1 \mathrm{~mm}$.

\section{DATA ANALYSIS}

The fMRI data analysis was done using Statistical Parametric Mapping 5 (SPM5, www.fil.ion.ucl.ac.uk/spm/). Pre-processing included realignment, slice timing, normalization to a standard EPI template, and smoothing with a 12-mm Gaussian kernel. Re-sampled voxel size (after normalization) was $3 \times 3 \times 3 \mathrm{~mm}$. For the first level model we convolved the onset of each single stimulation event as a stick function with the standard canonical hemodynamic response function used in SPM5. Rest periods were not explicitly modeled for. The rationale for this procedure (instead of modeling the whole stimulation period in an epoch related manner) was that detection power can be improved by such a model (Mechelli et al., 2003). Parameter images for the contrast "stimulation > (unmodeled) baseline" were generated for each subject and were then subjected to a second-level random effects analyses using (1) one-sample $t$-tests for testing main effects of stimulation in each group and each condition, (2) an independent sample $t$-test for between-group comparisons in each condition and (3) a $2 \times 2$ factorial design with group as a between-subject and type of stimulation as a within-subject factor in order to test for interactions between-group membership and type of stimulation. Global normalization was performed by including subjects' global activation level into the models as a covariate (ANCOVA). In order to provide a descriptive overview over the activation pattern of each group in each condition, we provide results of the one-sample $t$-tests with a threshold of $P<0.015$, uncorrected for the patient group and of $P<0.005$, uncorrected for the patient group (yielding comparable $\mathrm{T}$ thresholds for the given sample sizes). We then specifically tested for main effects of each stimulation paradigm in each group (one-sample $t$-tests), for group differences (two-sample $t$-tests) and for group $\times$ paradigm interactions (factorial design) in anatomical regions known to be relevant to olfactory processing (amygdala, thalamus, hippocampus Gottfried, 2006), and known to differentiate between Parkinson patients and control subjects (caudate, nucleus accumbens, anterior cingulate cortex, inferior frontal gyrus, Westermann et al., 2008). Masks for these regions of interest were generated using the Harvard-Oxford subcortical and cortical structural atlases distributed with FSL (FMRIB Analysis Group, University of Oxford, UK) with a population probability threshold of $50 \%$. For the ROI analyses, only results with a $P$ (family wise error, FWE-corrected for multiple comparisons across the search volumes [i.e., small volume corrected] $)<0.05$ are reported. 


\section{RESULTS}

\section{PSYCHOPHYSICAL OLFACTORY FUNCTION}

Parkinson's disease patients presented with significantly impaired olfactory function (TDI mean, 19.3; range, 11-31.5) compared to normal controls (TDI mean, 33.9; range, 25.25-38.5).

\section{RATINGS OF ODOR STIMULI PRESENTED DURING FMRI SCANS}

Idiopathic Parkinson's disease patients rated the stimuli as less intense while they rated both stimuli as more pleasant, whereas this tended to be the other way around for controls (interaction "group" * "ratings": $P=0.066$ ).

\section{ACTIVATION WITH ROSE-LIKE ODOR \\ Controls}

Rose-like odor stimulation in the control subjects yielded widespread activation of both subcortical and cortical areas largely consistent with previous studies on olfactory activation (Table 1). These included bilateral thalamus, amygdalae, hippocampi, midbrain, basal ganglia, and insular regions, as well as prefrontal and temporolateral regions. There was significant activation in all predefined regions of interest except right NAcc and left inferior frontal gyrus, pars triangularis (Figure 1A).

\section{Patients}

In contrast, patients did not show significant activations of the amydalae, hippocampi, and the thalamus. Instead the main foci of activation were in the striatum (caudate head), the superior medial prefrontal cortex, the anterior cingulate cortex, and lateral prefrontal cortices including the inferior frontal gyrus. In the ROI analysis, significantly less activation in patients compared to controls was observed in the right amygdala and in the right thalamus whereas significantly higher activation was found in the left inferior frontal gyrus, pars triangularis (Figure 1B; Table 2).

\section{ACTIVATION WITH THE SMELL OF ROTTEN EGGS $\left(\mathrm{H}_{2} \mathrm{~S}\right)$ Controls}

During stimulation with $\mathrm{H}_{2} \mathrm{~S}$ the main foci of activation were in the left hippocampus (activation maximum on whole brain level) and bilaterally in the amygdalae. Additionally, there was a significant positive BOLD signal in the left NAcc (Figure 1C).

\section{Patients}

In patients there were also significant activations in the left hippocampus and amygdala, whereas in the right amygdala activity was significantly reduced in comparison to the control group. There

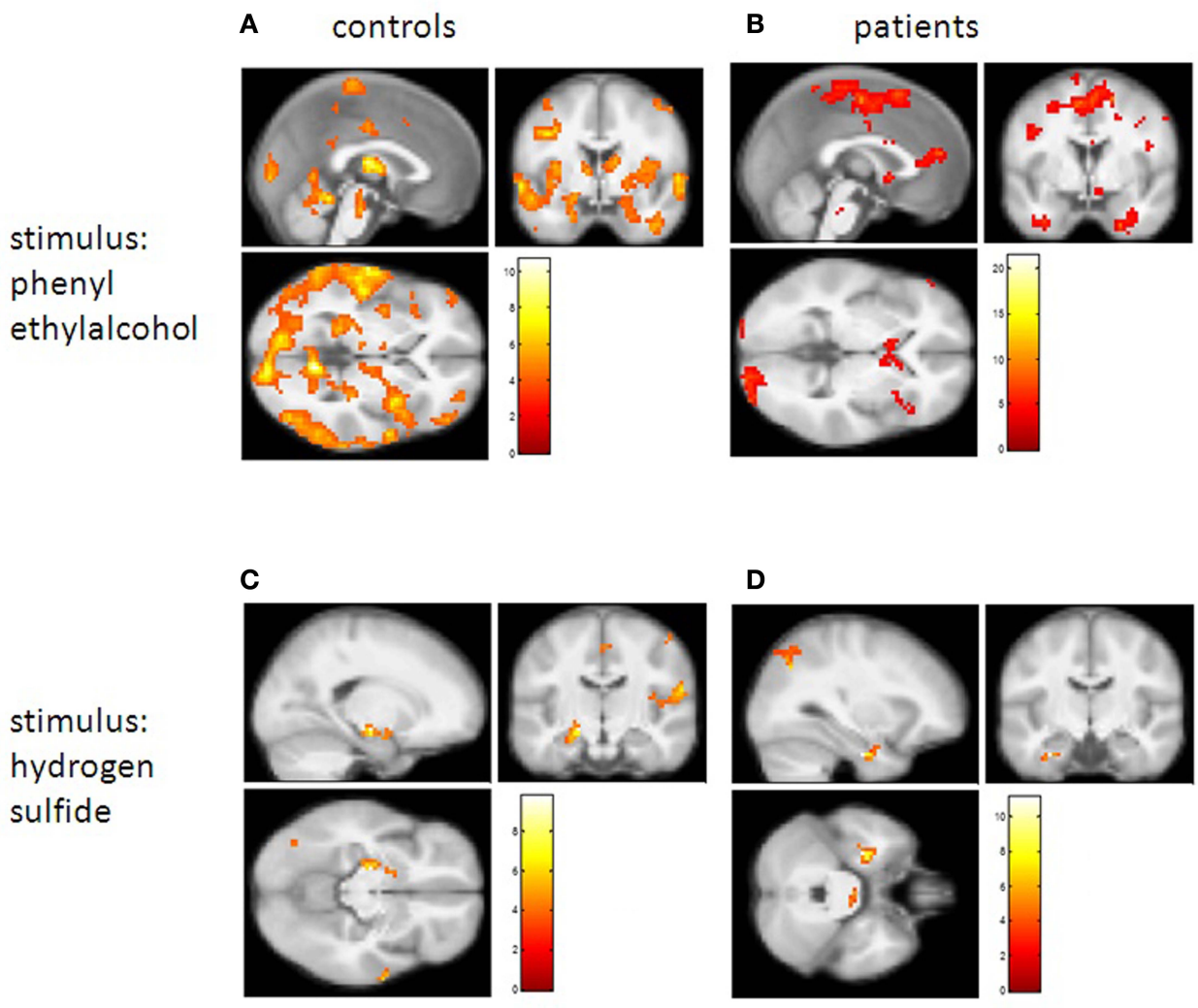

FIGURE 1 | Brain activation found in controls and patients. In the upper part results are shown for the pleasant rose odor, in the lower part results for the unpleasant $\mathrm{H}_{2} \mathrm{~S}$ are shown. For $\mathrm{A}$ and $\mathrm{B}$, sections are at MNI coordinates $x=0$, $y=0, z=0$. (A) Control group - positive BOLD responses to PEA stimulation (threshold $T=3.7, P=0.015$, uncorrected); (B) PD patients - positive BOLD responses to $P E A$ stimulation (threshold $T=3.7, P=0.005$, uncorrected);
(C) Control group - positive BOLD responses to hydrogen sulfate stimulation (threshold $T=3.7, P=0.015$, uncorrected), sections at MNI coordinates $x=-18$, $y=-15, z=-12$ (global peak voxel activation in the left hippocampus); (D) PD patients - positive BOLD responses to hydrogen sulfate stimulation (threshold $T=3.7, P=0.015$, uncorrected), sections at MNI coordinates $x=-30, y=-12$, $z=-30$ (global peak voxel activation in the left fusiform gyrus). 
Table 1 | Single group analyses.

\begin{tabular}{|c|c|c|c|c|c|c|}
\hline \multirow[t]{2}{*}{ Brain region } & \multicolumn{3}{|c|}{ MNI coordinates } & \multirow[t]{2}{*}{$N$} & \multirow[t]{2}{*}{$T$} & \multirow[t]{2}{*}{$\boldsymbol{P}$} \\
\hline & $x$ & $y$ & $z$ & & & \\
\hline \multicolumn{7}{|l|}{ PEA CONTROLS } \\
\hline Left middle temporal gyrus & -57 & -57 & 15 & 8526 & 10.67 & $<0.001$ \\
\hline Right middle frontal gyrus & 54 & 12 & 48 & 123 & 6.12 & $<0.001$ \\
\hline Right inferior temporal gyrus & 45 & 3 & -36 & 57 & 6.04 & $<0.001$ \\
\hline Right superior temporal gyrus & 42 & -42 & 6 & 19 & 6.03 & $<0.001$ \\
\hline Left paracentral & -9 & -39 & 72 & 134 & 5.60 & $<0.001$ \\
\hline Right middle orbitofrontal cortex & 33 & 51 & -6 & 191 & 5.49 & $<0.001$ \\
\hline Right middle cingulate gyrus & 3 & -15 & 39 & 50 & 5.27 & $<0.001$ \\
\hline Left posterior cingulate gyrus & -6 & -45 & 21 & 38 & 5.26 & $<0.001$ \\
\hline Left superior frontal gyrus & -27 & 57 & 21 & 39 & 5.16 & $<0.001$ \\
\hline Left postcentral gyrus & -39 & -39 & 66 & 9 & 4.80 & $<0.001$ \\
\hline Right precuneus & 6 & -39 & 54 & 29 & 4.78 & $<0.001$ \\
\hline Left precentral gyrus & -15 & -27 & 60 & 13 & 4.47 & $<0.001$ \\
\hline Left middle frontal gyrus & -42 & 51 & 0 & 10 & 4.32 & 0.001 \\
\hline Left middle cingulate gyrus & 3 & 12 & 42 & 9 & 4.32 & 0.001 \\
\hline \multicolumn{7}{|l|}{ PATIENTS } \\
\hline Right precentral gyrus & 24 & -30 & 72 & 89 & 21.50 & $<0.001$ \\
\hline Left postcentral gyrus & -24 & -42 & 42 & 142 & 15.90 & $<0.001$ \\
\hline \multirow[t]{3}{*}{ Left superior frontal gyrus } & -15 & 48 & 39 & 82 & 13.42 & $<0.001$ \\
\hline & -27 & -9 & 51 & 66 & 13.30 & $<0.001$ \\
\hline & -6 & -3 & 51 & 563 & 12.11 & $<0.001$ \\
\hline \multirow[t]{2}{*}{ Right superior occipital gyrus } & 24 & -84 & 18 & 256 & 11.04 & $<0.001$ \\
\hline & 48 & -39 & 27 & 44 & 10.67 & $<0.001$ \\
\hline Right middle frontal gyrus & 45 & 21 & 33 & 53 & 10.04 & $<0.001$ \\
\hline Right fusiform gyrus & 30 & -3 & -36 & 30 & 9.96 & $<0.001$ \\
\hline Left anterior cingulate gyrus & -3 & 48 & 15 & 26 & 9.96 & $<0.001$ \\
\hline \multirow[t]{2}{*}{ Right caudate } & 6 & 12 & -3 & 33 & 9.76 & $<0.001$ \\
\hline & 12 & -48 & 75 & 18 & 9.66 & $<0.001$ \\
\hline
\end{tabular}

was reduced activity (compared to baseline) during stimulation bilaterally in the caudate head. Compared to the control group, activity both in the caudate heads and in the NAcc was lower in the patient than in the control group (Figure 1D).

\section{Interactions}

There were significant group $\times$ stimulation type interactions in the striatum (caudate heads bilaterally and left NAcc) and in the left inferior frontal gyrus, pars triangularis. In the striatum (Figure 2A shows the results for the left caudate head as an example) controls did not show differential responses to the two stimulus types, whereas there was a highly significant difference in the control group with activation during stimulation with the pleasant odor and deactivation during stimulation with the unpleasant odor. In the left inferior frontal gyrus the significant interaction resulted from a highly positive response to the pleasant odor in the patients group which was not present for the unpleasant odor and for both odors in the control group (Figure 2).

\section{DISCUSSION}

In the present study we compared the effects of pleasant (PEA) and unpleasant $\left(\mathrm{H}_{2} \mathrm{~S}\right)$ olfactory stimulation on brain activity in patients with PD and healthy controls. We observed a striking

\begin{tabular}{|c|c|c|c|c|c|c|}
\hline \multirow[t]{2}{*}{ Brain region } & \multicolumn{3}{|c|}{ MNI coordinates } & \multirow[t]{2}{*}{$N$} & \multirow[t]{2}{*}{$T$} & \multirow[t]{2}{*}{$P$} \\
\hline & $x$ & $y$ & $z$ & & & \\
\hline & -42 & -30 & 60 & 134 & 9.15 & $<0.001$ \\
\hline & 45 & -24 & 51 & 34 & 9.05 & $<0.001$ \\
\hline Left middle occipital gyrus & -45 & -84 & 18 & 26 & 8.75 & $<0.001$ \\
\hline Left superior occipital gyrus & -15 & -78 & 42 & 71 & 8.40 & $<0.001$ \\
\hline Right superior temporal pole & 45 & 15 & -21 & 9 & 8.29 & $<0.001$ \\
\hline Right rolandic operculum & 51 & -18 & 15 & 12 & 8.18 & $<0.001$ \\
\hline Right superior parietal gyrus & 21 & -54 & 51 & 26 & 7.81 & $<0.001$ \\
\hline \multirow[t]{2}{*}{ Left fusiform gyrus } & -30 & -9 & -33 & 34 & 7.45 & $<0.001$ \\
\hline & 33 & 24 & -12 & 22 & 7.44 & $<0.001$ \\
\hline Right inferior frontal gyrus & 45 & 27 & 12 & 17 & 6.70 & $<0.001$ \\
\hline Left superior temporal pole & -51 & 12 & -12 & 15 & 6.46 & $<0.001$ \\
\hline Left precentral gyrus & -45 & 3 & 30 & 10 & 6.03 & $<0.001$ \\
\hline \multicolumn{7}{|l|}{$\mathrm{H}_{2} \mathrm{~S}$ CONTROLS } \\
\hline Left hippocampus & -18 & -15 & -12 & 71 & 9.80 & 0.004 \\
\hline Right rolandic operculum & 60 & -18 & 15 & 186 & 7.24 & $<0.001$ \\
\hline Right posterior cingulate gyrus & 9 & -42 & 12 & 55 & 5.90 & $<0.001$ \\
\hline Right middle temporal gyrus & 66 & -6 & -12 & 18 & 5.88 & $<0.001$ \\
\hline Left fusiform gyrus & -33 & -72 & -15 & 19 & 5.07 & $<0.001$ \\
\hline Left superior frontal gyrus & 0 & -12 & 48 & 16 & 5.05 & $<0.001$ \\
\hline Right postcentral gyrus & 51 & -18 & 57 & 9 & 4.44 & 0.001 \\
\hline \multicolumn{7}{|l|}{ PATIENTS } \\
\hline Left fusiform gyrus & -30 & -12 & -30 & 37 & 11.16 & $<0.001$ \\
\hline Right inferior occipital gyrus & 51 & -72 & -15 & 32 & 8.45 & $<0.001$ \\
\hline Left parahippocampal gyrus & -12 & 3 & -18 & 16 & 7.57 & $<0.001$ \\
\hline Left angular gyrus & -36 & -69 & 42 & 71 & 7.30 & $<0.001$ \\
\hline Right middle temporal pole & 24 & 12 & -39 & 17 & 6.74 & $<0.001$ \\
\hline Left parahippocampal gyrus & 0 & -18 & -30 & 10 & 5.47 & 0.001 \\
\hline Right inferior temporal gyrus & 57 & -33 & -18 & 9 & 4.74 & 0.002 \\
\hline Right putamen & 21 & 15 & -6 & 25 & 4.72 & 0.002 \\
\hline
\end{tabular}

difference in odor processing between PD patients and healthy controls depending on the affective tone of the presented odors. When applying a pleasant odor, brain regions were activated in both groups that are typically involved in olfactory processing (amygdala, hippocampus, thalamus) (Gottfried, 2006), but consistent with the literature, there was an asymmetric (right-sided) reduction of activation in PD patients in the amygdala and the thalamus which may relate to a lack of responsiveness of amygdala in PD patients to emotional stimuli (Yoshimura et al., 2005). Conversely, patients showed consistent and strong activation in the ventral striatum and in prefrontal lateral areas, yielding a significant difference compared to the control group in the pars triangularis of the left inferior frontal gyrus. This finding is in line with the assumption of an upregulation of dopaminergic responses to pleasant odor in PD patients. By introducing an unpleasant stimulus in our experimental design we are able to show that the effect in these dopaminergic areas depends critically on the hedonic value of the odor. For the unpleasant stimuli there were decreases in ventral striatal activations and there were no increases of activation in prefrontal lateral regions. Again, as for PEA stimulation, there was reduced activity in the right amygdalo-hippocampal complex. 


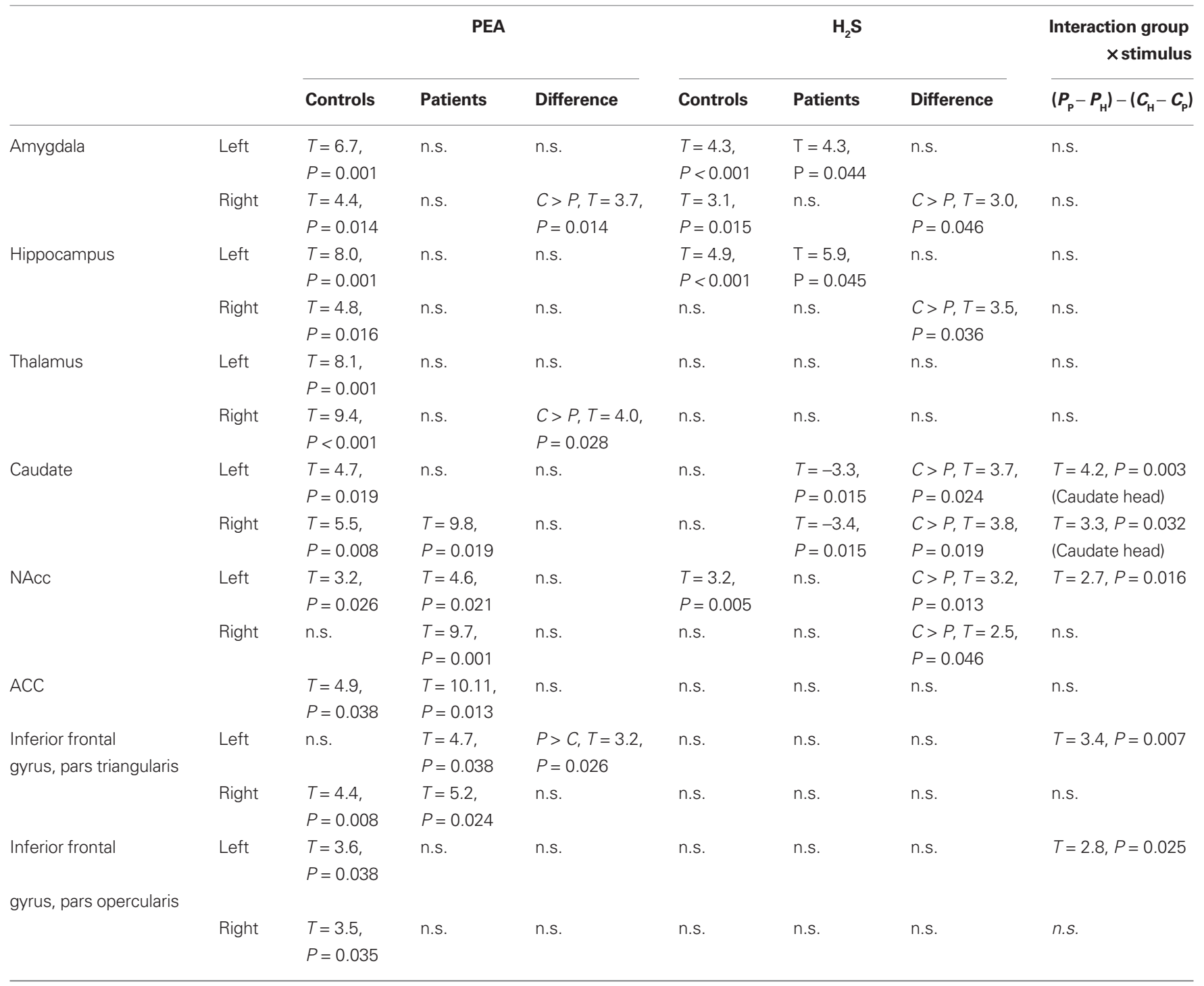

Together with the behavioral results, these findings suggest that there is a dissociation of intensity and valence processing and that both components are differentially affected in hyposmic PD patients. While reduced intensity perception might be reflected by reduced activation of primary relay structures of the central nervous olfaction system (such as the amygdala), changes in valence perception might be attributed to changes in activation of the ventral striatum and left prefrontal areas.

In a series of studies Royet et al. $(1999,2001,2003)$ have shown that different olfactory tasks correspond with selective activation of different brain areas; depending on the type of olfactory task, aspects of odor processing were lateralized. A greater right-sided than leftsided activity in the orbitofrontal cortex (OFC) was observed during exposure to odorants with strong affective valence under a familiarity judgment task (Zald and Pardo, 1997, 2000; Royet et al., 2000). Left OFC activity however, was significantly higher during ratings of the hedonic character of odors. Therefore, the lateralization of activation in the OFC depended on whether the task required odor recognition or a valence judgment (Royet et al., 2001). In the present study we used only two odors which did not sufficiently allow us to assess activation in relation to graded judgments across the entire scale of possible ratings. We also did not manipulate task demands during fMRI scanning. However, in our study the combination of fMRI data and ratings suggests that the different patterns of activation in PD patients and controls were related to differences in the evaluation of intensity and valence. Hyposmic PD patients rated the two stimuli as less intense, but also as more pleasant, whereas this tended to be the other way around for controls. This is in line with the stronger activation of the ventral striatum and the left inferior frontal cortex in PD patients. In contrast, following exposure to $\mathrm{H}_{2} \mathrm{~S}$ controls showed reduced activation of the ventral striatum. In controls, a significant bilateral activation in the amygdalo-hippocampal complex was observed which supports the results of Anderson et al. (2003) (but see also Winston et al., 2005). They found amygdala activation to be strongly associated with, the intensity of odors, which is in line with the results of our study. 

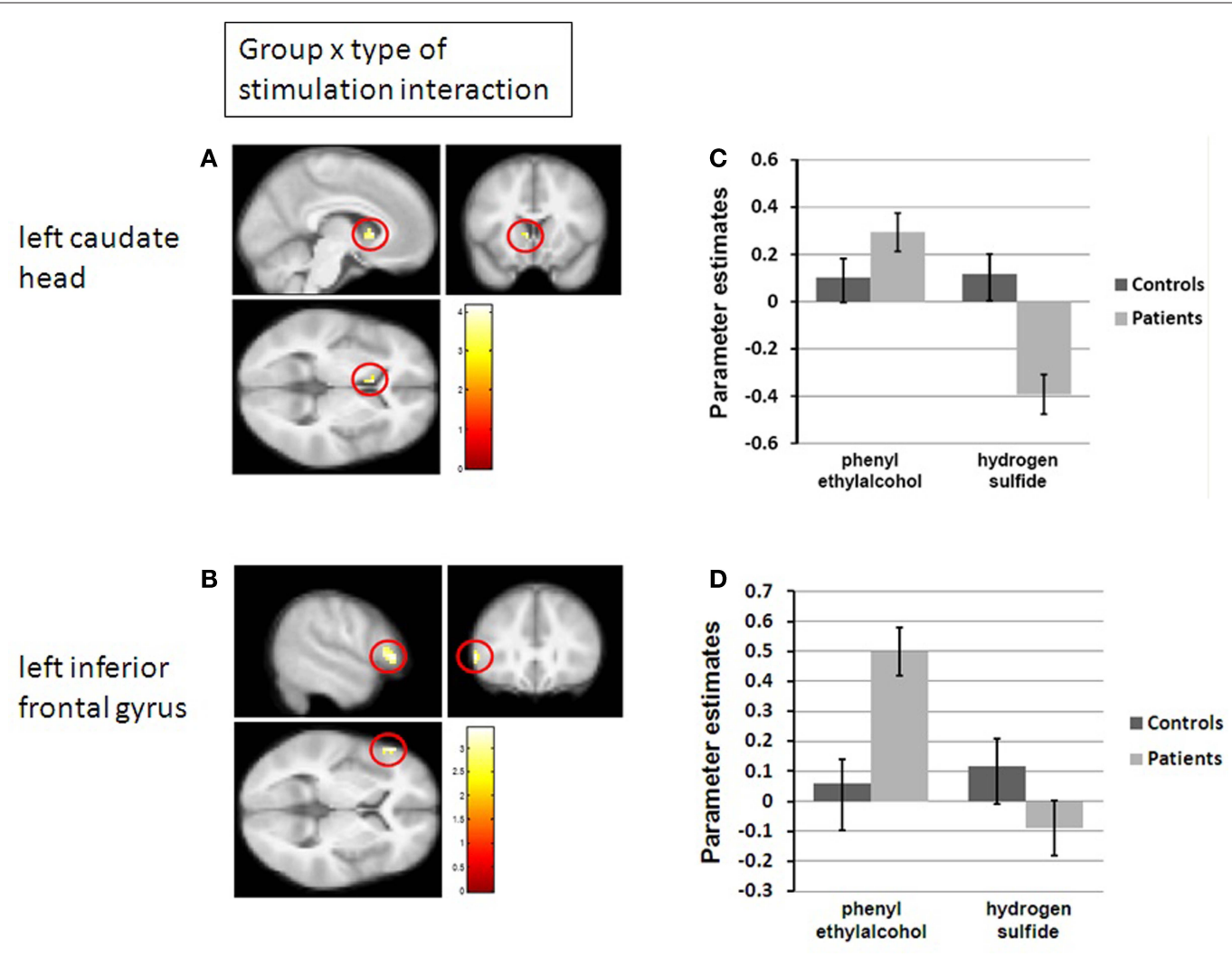

FIGURE 2 | Differential brain activation in controls and patients.

(A) Group $\times$ type of stimulation interaction in the left caudate head $(P<0.05$, FWE-corrected for search volume left caudate). MNI coordinates of peak voxel: $x=-6, y=15, z=3$. (B) Group $\times$ type of stimulation interaction in the left inferior frontal gyrus $(P<0.05$, FWE-corrected for search volume left inferior frontal gyrus, pars triangularis). MNI coordinates of peak voxel: $x=-54, y=30, z=3$. (C,D) Parameter estimates separately for patients and controls, both for the pleasant PEA and the unpleasant $\mathrm{H}_{2} \mathrm{~S}$, corresponding to $\mathbf{A}$ and $\mathbf{B}$, respectively.
An important difference between the present study and previous work by Westermann et al. (2008) relates to the side of stimulation. However, although Westermann et al. stimulated one nostril only, while in our study both nostrils were stimulated, both studies revealed lateralized activation in response to the odorous stimuli, indicating that these side differences might be a specific finding in PD patients. On the other hand, the differences in stimulation may help to explain other differences between activated areas found in the two studies.

The study is partly limited by the relatively small number of investigated subjects, which calls for studies in larger groups of patients. Another weakness of the study was the missing assessment of dementia. However, none of the patients appeared to be suffering from dementia, as all of them were able to perform the required tasks - quite demanding tasks for the psychophysical measurements of olfactory function, which should have unveiled the presence of major cognitive deficits. In addition, while possible differences between patients and controls in terms of dementia cannot be excluded, it appears unlikely that dementia would produce lateralized differences in the processing of odors.

To summarize, alterations in olfactory processing in PD appear to produce a distinct pattern of cortical and subcortical activation compared to healthy individuals. This may partly explain why the experience - defined in terms of two primary dimensions of odors, intensity and valence - may vary between PD patients and healthy controls.

\section{ACKNOWLEDGEMENT}

We would like to thank Reesa Grushka for her invaluable help in language editing.

\section{REFERENCES}

Anderson, A. K., Christoff, K., Stappen, I., Panitz, D., Ghahremani, D. G., Glover, G., Gabrieli, J. D., and Sobel, N. (2003). Dissociated neural representations of intensity and valence in human olfaction. Nat. Neurosci. 6, 196-202.
Barz, S., Hummel, T., Pauli, E., Majer, M., Lang, C. J., and Kobal, G. (1997). Chemosensory event-related potentials in response to trigeminal and olfactory stimulation in idiopathic Parkinson's disease. Neurology 49, 1424-1431.

Beck, A. T., Ward, C. M., Mendelson, M., Mock, J. E., and Erbaugh, J. K.
(1961). An inventory for measuring depression. Arch. Gen. Psychiatry 4, 561-571.

Berendse, H. W., and Ponsen, M. M. (2006). Detection of preclinical Parkinson's disease along the olfactory trac(t). J. Neural Transm. Suppl. 321-325.
Croy, I., Lange, K., Krone, F., Negoias, S., Seo, H. S., and Hummel, T. (2009). Comparison between odor thresholds for phenyl ethyl alcohol and butanol. Chem. Senses 34, 523-527.

Daniel, S. E., and Lees, A. J. (1993). Parkinson's disease Society Brain Bank, London: overview and 
research. J. Neural. Transm. Suppl. 39, 165-172.

Doty, R. L., Bromley, S. M., and Stern, M. B. (1995). Olfactory testing as an aid in the diagnosis of Parkinson's disease: development of optimal discrimination criteria. Neurodegeneration 4, 93-97.

Doty, R. L., Deems, D., and Steller, S. (1988). Olfactory dysfunction in Parkinson's disease: a general deficit unrelated to neurologic signs, disease stage, or disease duration. Neurology $38,1237-1244$.

Fahn, S., and Elton, R.L. (1987)."Members of the UPDRS Development Committee Unified Parkinson Rating Scale," in: Recent Developments in Parkinson's Disease, eds S. Fahn, C. D. Marsden, D. B. Calne, and M. Goldstein (New York: Macmillan Publishing), 153-163.

Gottfried,J.A. (2006). Smell: central nervous processing. Adv. Otorhinolaryngol. $63,44-69$.

Haehner, A., Hummel, T., Hummel, C., Sommer, U., Junghanns, S., and Reichmann, H. (2007). Olfactory loss may be first sign of idiopathic Parkinson's disease. Mov. Disord. 22, 839-842.

Hautzinger, M., Bailer, M., Worall, H., and Keller, F. (1995). Beck-DepressionsInventar (BDI). Göttingen: Hogrefe.

Hawkes, C. H., and Shephard, B. C. (1998). Olfactory evoked responses and identification tests in neurological disease. Ann. N. Y. Acad. Sci. 855, 608-615.

Hummel, T., Kobal, G., Gudziol H., and Mackay-Sim, A. (2007). Normative data for the "Sniffin' Sticks" including tests of odor identification, odor discrimination, and olfactory thresh- olds: an upgrade based on a group of more than 3,000 subjects. Eur. Arch. Otorhinolaryngol. 264, 237-243.

Hummel, T., Kobal, G., and Mokrusch, T. (1993). "Chemosensory evoked potentials in patients with Parkinson's disease," in New Developments in EventRelated Potentials, eds H. J. Heinze, T. Münte, and G. R. Mangun (Boston: Birkhäuser Verlag), 275-281.

Hummel, T., Sekinger, B., Wolf, S., Pauli, E., and Kobal, G. (1997). "Sniffin" Sticks": olfactory performance assessed by the combined testing of odor identification, odor discrimination and olfactory threshold. Chem. Senses 22, 39-52.

Hummel, T., Witt, M., Reichmann, H., Welge-Luessen, A., and Haehner, A. (2010). Immunohistochemical, volumetric, and functional neuroimaging studies in patients with idiopathic Parkinson's disease. J. Neurol. Sci. 289, 119-122.

Kobal, G., Hummel, T., Sekinger, B., Barz, S., Roscher, S., and Wolf, S. R. (1996). "Sniffin' Sticks": screening of olfactory performance. Rhinology 34, 222-226. Litvan, I., Bhatia, K.P., Burn, D. J., Goetz, C. G., Lang, A. E., McKeith, I., Quinn, N., Sethi, K. D., Shults C., and Wenning, G. K. (2003). Society Scientific Issues Committee report: SIC Task Force appraisal of clinical diagnostic criteria for Parkinsonian disorders. Mov. Disord. 18, 467-486.

Mechelli, A., Henson, R. N., Price, C. J., and Friston, K. J. (2003). Comparing event-related and epoch analysis in blocked design fMRI. Neuroimage 18, 806-810.

Ramaker,C., Marinus, J., Stiggelbout,A.M., and Van Hilten, B. J. (2002). Systematic evaluation of rating scales for impairment and disability in Parkinson's disease. Mov. Disord. 17, 867-876.

Royet, J. P., Hudry, J., Zald, D. H., Godinot, D., Gregoire, M. C., Lavenne, F., Costes, N., and Holley, A. (2001). Functional neuroanatomy of different olfactory judgments. Neuroimage 13, 506-519.

Royet, J. P., Koenig, O., Gregoire, M. C., Cinotti, L., Lavenne, F., Le Bars, D., Costes, N., Vigouroux, M., Farget, V., Sicard, G., Holley, A., Mauguiere, F., Comar, D., and Froment, J. C. (1999). Functional anatomy of perceptual and semantic processing for odors. J. Cogn. Neurosci. 11, 94-109.

Royet, J. P., Plailly, J., Delon-Martin, C., Kareken, D. A., and Segebarth, C. (2003). fMRI of emotional responses to odors: influence of hedonic valence and judgment, handedness, and gender. Neuroimage 20, 713-728.

Royet, J. P., Zald, D., Versace, R., Costes, N., Lavenne, F., Koenig, O., and Gervais, R. (2000). Emotional responses to pleasant and unpleasant olfactory, visual, and auditory stimuli: a positron emission tomography study.J. Neurosci. 20, 7752-7759.

Westermann, B., Wattendorf, E., Schwerdtfeger, U., Husner, A., Fuhr, P., Gratzl, O., Wolfensberger, M., Hummel, T., Bilecen, D., and WelgeLüssen, A. (2008). Functional imaging of the cerebral olfactory system in patients with Parkinson's disease. J. Neurol. Neurosurg. Psychiatry 79, 19-24.

Winston, J. S., Gottfried, J. A., Kilner, J. M., and Dolan, R. J. (2005). Integrated neural representations of odor intensity and affective valence in human amygdala. J. Neurosci. 28, 8903-8907.
Yoshimura, N., Kawamura, M., Masaoka, Y., and Homma, I. (2005). The amygdala of patients with Parkinson's disease is silent in response to fearful facial expressions. Neuroscience 131, 523-534.

Zald, D. H., and Pardo, J. V. (1997). Emotion, olfaction, and the human amygdala: amygdala activation during aversive olfactory stimulation. Proc. Natl. Acad. Sci. U.S.A. 15, 4119-4124.

Zald, D. H., and Pardo, J. V. (2000). Functional neuroimaging of the olfactory system in humans. Int. J. Psychophysiol. 36, 165-181.

Conflict of Interest Statement: The authors declare that the research was conducted in the absence of any commercial or financial relationships that could be construed as a potential conflict of interest.

Received: 30 April 2010; accepted: 27 September 2010; published online: 28 October 2010.

Citation: Hummel T, Fliessbach K, Abele M, Okulla T, Reden J, Reichmann H, Wüllner U and Haehner A (2010). Olfactory fMRI in patients with Parkinson's disease. Front. Integr. Neurosci. 4:125. doi: 10.3389/ fnint.2010.00125

Copyright (c) 2010 Hummel, Fliessbach, Abele, Okulla, Reden, Reichmann, Wülner and Haehner. This is an openaccess article subject to an exclusive license agreement between the authors and the Frontiers Research Foundation, which permits unrestricted use, distribution, and reproduction in any medium, provided the original authors and source are credited. 